OPEN ACCESS

Edited by:

Thomas Leonhard Stöggl, University of Salzburg, Austria

Reviewed by:

Beat Knechtle,

University of Zurich, Switzerland

Patrick Wahl,

German Sport University Cologne,

Germany

*Correspondence:

Peter Hofmann

peter.hofmann@uni-graz.at

Specialty section:

This article was submitted to

Exercise Physiology,

a section of the journal

Frontiers in Physiology

Received: 28 October 2016 Accepted: 08 May 2017

Published: 24 May 2017

Citation:

Hofmann P and Tschakert G (2017) Intensity- and Duration-Based Options to Regulate Endurance Training.

Front. Physiol. 8:337.

doi: 10.3389/fphys.2017.00337

\section{Intensity- and Duration-Based Options to Regulate Endurance Training}

\author{
Peter Hofmann * and Gerhard Tschakert \\ Exercise Physiology, Training and Training Therapy Research Group, Institute of Sports Science, University of Graz, Graz, \\ Austria
}

The regulation of endurance training is usually based on the prescription of exercise intensity. Exercise duration, another important variable of training load, is rarely prescribed by individual measures and mostly set from experience. As the specific exercise duration for any intensity plays a substantial role regarding the different kind of cellular stressors, degree, and kind of fatigue as well as training effects, concepts integrating the prescription of both intensity and duration within one model are needed. An according recent approach was the critical power concept which seems to have a physiological basis; however, the mathematical approach of this concept does not allow applying the three zones/two threshold model of metabolism and its different physiological consequences. Here we show the combination of exercise intensity and duration prescription on an individual basis applying the power/speed to distance/time relationship. The concept is based on both the differentiation of intensities by two lactate or gas exchange variables derived turn points, and on the relationship between power (or velocity) and duration (or distance). The turn points define three zones of intensities with distinct acute metabolic, hormonal, and cardio-respiratory responses for endurance exercise. A maximal duration exists for any single power or velocity such as described in the power-duration relationship. Using percentages of the maximal duration allows regulating fatigue, recovery time, and adaptation for any single endurance training session. Four domains of duration with respect to induced fatigue can be derived from maximal duration obtained by the power-duration curve. For any micro-cycle, target intensities and durations may be chosen on an individual basis. The model described here is the first conceptual framework of integrating physiologically defined intensities and fatigue related durations to optimize high-performance exercise training.

Keywords: exercise prescription, intensity, duration, endurance exercise, athletes

\section{INTRODUCTION}

The regulation of endurance training is usually based on the prescription of individual exercise intensity zones/domains, (Meyer et al., 2005; Pescatello, 2014, p. 168) whereas, in contrast, exercise duration is rarely prescribed by individual measures and mostly set from personal experience or "usual" settings. Tremblay et al. (2005) critically mentioned that little research tempting to isolate the effect of exercise duration has been done but they suggested a duration threshold for hormonal responses especially for low intensity exercise. This is in line with earlier results 
by Viru et al. (1996) proposing that in exercise performed below a certain threshold intensity, hormonal responses will only occur after a certain long duration. As the hormonal changes trigger acute and chronic adaptation it is suggested that not only intensity but also the duration for any specific intensity is crucial to induce training effects or to avoid overload (Viru, 1995, p. 1-20). Recently, it was shown by Skovgaard et al. (2016) that muscle PGC-1 $\alpha$ mRNA, identified as a key regulator of mitochondrial biogenesis and oxidative genes, did not change significantly after $60 \mathrm{~min}$ of endurance exercise in their study. This short duration and a high fitness level did not allow to sufficiently challenge muscle PGC- $1 \alpha$ mRNA for the relative low exercise intensity $\left(60 \%\right.$ of $\left.\mathrm{VO} 2_{\max }\right)$ applied. However, including high intensity speed endurance exercise provided a stimulus for muscle mitochondrial biogenesis, substrate regulation, and angiogenesis.

Consequently, concerning training effects (Platonov, 1999; Noakes, 2000; Abbiss and Laursen, 2005), concepts integrating the prescription of both intensity and duration within one model are needed with respect to the main aims in endurance training which are to increase maximal oxygen uptake, the maximal sustainable speed, or power (threshold speed), and to increase economy and time to exhaustion (Lundby and Robach, 2015).

Several authors prescribed the distribution of various intensity domains for endurance training (Esteve-Lanao et al., 2005; Seiler and Kjerland, 2006; Seiler, 2010; Stöggl and Sperlich, 2015), but explicit prescriptions of an optimal duration for each individual intensity domain are still missing. Pettitt (2016) recently combined exercise intensity and duration by introducing a critical velocity similar to the critical power concept (Vanhatalo et al., 2011; Poole et al., 2016). The CP model itself is not based on physiological measures, although it seems to have a physiological basis which was shown to be related to the maximal lactate steady state intensity (Jones et al., 2008, 2010). This concept however, does not include a differentiation of all intensity domains (Meyer et al., 2005; Hofmann and Tschakert, 2010) which are known to trigger specific acute physiological responses, which are suggested to be crucial for a successful training adaptation (Hoppeler, 2016). Dekerle et al. (2003) as well as Pringle and Jones (2002) showed that the critical power calculated from a given range of exhaustion time did not correspond to the maximal lactate steady state (mLaSS) similar to Brickley et al. (2002) indicating the need to combine both aspects into one model recently shown by Burnley and Jones (2016).

It is well-prescribed that competitive endurance athletes using the polarization model train up to 13 training sessions per week with an intensity distribution of about $80 \%$ of total training volume performed at low intensity and about $20 \%$ high-intensity work such as interval training (Esteve-Lanao et al., 2005; Seiler and Kjerland, 2006; Seiler, 2010; Stöggl and Sperlich, 2015). From this point of view, a focus on optimization of both the low intensity-high volume and the high intensity-low volume parts of the training as well as concepts and models to prescribe both intensity and duration including physiologically relevant zones are required. Aim of the paper is to give a theoretical framework prescribing both intensity and duration for endurance training.

\section{PRESCRIPTION OF INTENSITY}

The prescription of exercise intensity for endurance-type exercise is usually based on exercise markers from maximal and/or sub-maximal incremental exercise tests (Meyer et al., 2005). Guidelines recommend using percentages of maximal oxygen uptake $\left(\mathrm{VO}_{2 \max }\right)$, maximal heart rate $\left(\mathrm{HR}_{\max }\right)$, or maximal power output $\left(\mathrm{P}_{\max }\right)$ for setting exercise intensity (Pescatello, 2014, p. 168). However, threshold or turn point concepts are suggested to be the gold standard for exercise intensity prescription in practice (Meyer et al., 2005) although still critically discussed (Mann et al., 2013). Actually, most authors agree to set training intensities by a three phase and two threshold model (Meyer et al., 2005; Hofmann and Tschakert, 2010) indicated by a first lactate $\left(\mathrm{LT}_{1} / \mathrm{LTP}_{1}\right)$ or ventilatory $\left(\mathrm{VT}_{1}\right)$ and a second lactate $\left(\mathrm{LT}_{2} / \mathrm{LTP}_{2}\right)$ or ventilatory $\left(\mathrm{VT}_{2}\right)$ threshold or turn point which has been successfully integrated into the practice (Seiler and Kjerland, 2006; Seiler, 2010; Algrøy et al., 2011; Muñoz et al., 2014a,b; Tønnessen et al., 2014, 2015). Figure 1 shows an example of the time course of selected variables and the according turn points $\mathrm{LTP}_{1} / \mathrm{VT}_{1}$ and $\mathrm{LTP}_{2} / \mathrm{VT}_{2}$ for a trained cyclist.

Several variables enable to discern three distinct phases of metabolism and cardio-respiratory responses which allow setting defined intensities for continuous or interval-type exercise (Hofmann and Tschakert, 2010; Tschakert and Hofmann, 2013). According to the lactate shuttle theory (Brooks, 1986, 2009) the first lactate turn point $\left(\mathrm{LTP}_{1}\right)$ is defined as the first increase in blood lactate concentration (La) accompanied by a first change of increase in ventilation $\left(\mathrm{VT}_{1}\right)$ and distinct changes in other ventilatory variables. The second lactate turn point $\left(\mathrm{LTP}_{2}\right)$ is defined as the second abrupt increase in La accompanied by a sharp increase in ventilation $\left(\mathrm{VT}_{2}\right)$ and distinct changes in other ventilatory variables (Figures $\mathbf{1}, \mathbf{2}$ ). It has to be mentioned that the chosen incremental test protocol influences the accuracy of any threshold determination and the validity to prescribe constant load or intermittent-type exercise. A careful choice of the protocol is accordingly substantial. A detailed discussion of this problem, however, is not within the scope of this article but discussed elsewhere (McLellan, 1985; Amann et al., 2004).

\section{Continuous Exercise}

The first and the second turn points are sub-maximal markers from incremental exercise which can be used to prescribe defined exercise workloads with distinct and defined metabolic, cardiorespiratory, and hormonal responses as shown recently by our working group for constant load and matched intermittenttype exercise (Tschakert and Hofmann, 2013; Moser et al., 2015; Tschakert et al., 2015). During exercise below LTP $_{1}$, no increase in La above baseline level was detected for constant load exercise, and it was shown recently that this intensity can be sustained for a very long duration of up to $24 \mathrm{~h}$ in trained ultra-distance athletes (Pokan et al., 2014). Increasing the workload above LTP $_{1}$ leads to an increase in La above baseline, but after several minutes a La steady state is built up. The mLaSS is reached at $\mathrm{LTP}_{2}$ power output, but this intensity is clearly limited in time (Dittrich et al., 2014) but independent of exercise mode (Fontana et al., 2009), training status, and temperature (Périard et al., 2012). Although 

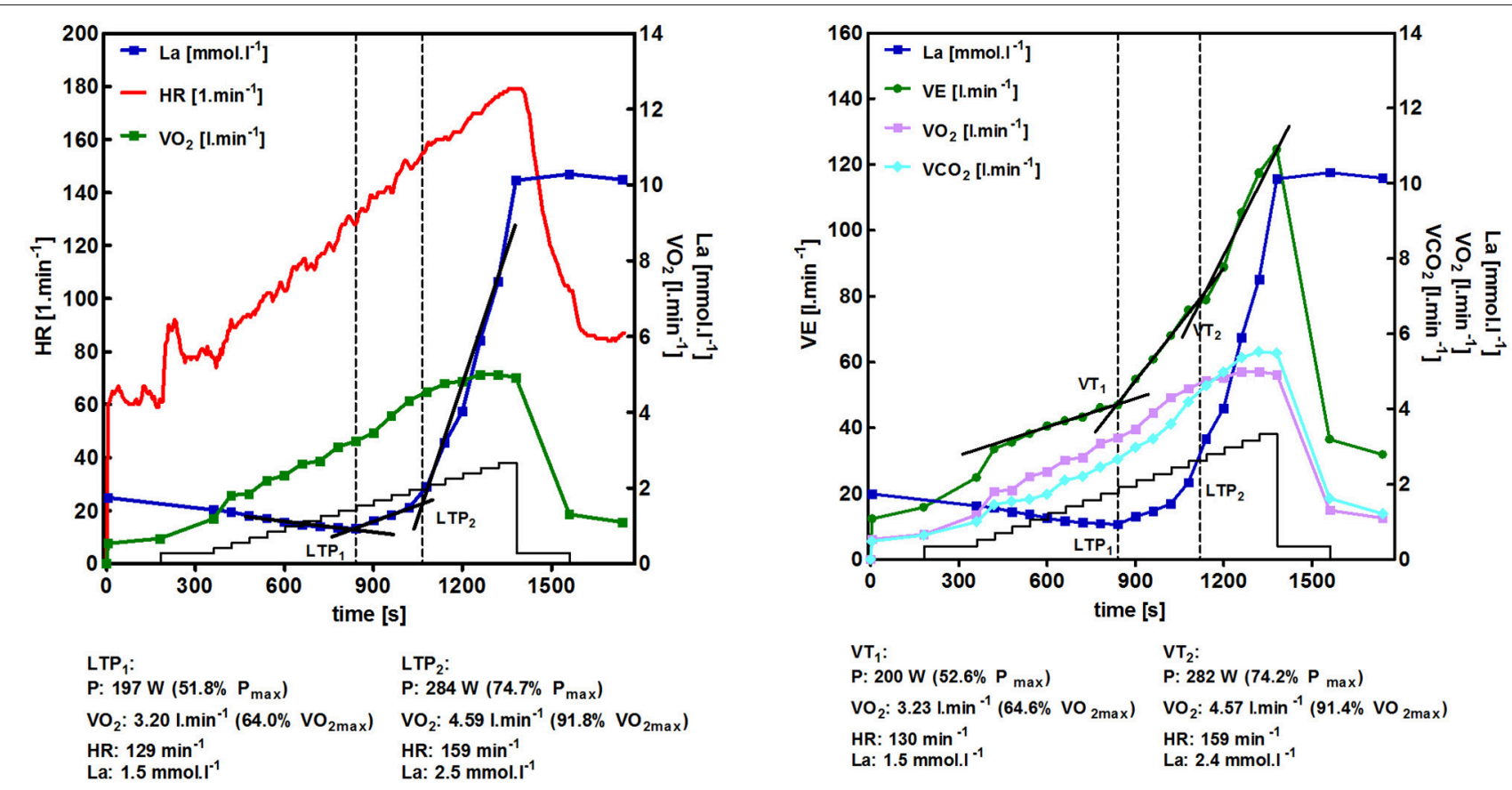

FIGURE 1 | Time course of heart rate (HR), oxygen uptake $\left(\mathrm{VO}_{2}\right)$, and lactate $(\mathrm{La})$ as well as the first (LTP 1 ) and the second $($ LTP 2$)$ lactate turn point during an incremental cycle ergometer exercise test in a well-trained cyclist (A). Time course of ventilation (VE), oxygen uptake (VO⿰亻 2 ), carbon dioxide output $\left(\mathrm{VCO}_{2}\right)$, and lactate $(\mathrm{La})$ as well as the first $\left(\mathrm{VT}_{1}\right)$ and the second $\left(\mathrm{VT}_{2}\right)$ ventilatory turn point during incremental cycle ergometer exercise in a well-trained cyclist (B).

mLaSS intensity can be determined rather precisely, time to exhaustion at the mLaSS still can vary distinctively between athletes. Faude et al. (2017) showed a low reliability of time-toexhaustion and blood lactate concentration at mLaSS indicating that a precise individual prescription of exercise still remains challenging especially with respect to duration.

Workloads above $\mathrm{LTP}_{2} / \mathrm{VT}_{2}$ lead to a constant increase in $\mathrm{La}$ until the individual level of tolerance has been reached. This is also reflected in the response of adrenaline and noradrenaline (Moser et al., 2015). Figure 3 shows schematically the time course of La for the three different exercise intensity zones. Percentages of maximal heart rate $\left(\% \mathrm{HR}_{\max }\right)$, of maximal oxygen uptake $\left(\% \mathrm{VO}_{2 \max }\right)$, or $\% \mathrm{HR}$ reserve $(\% \mathrm{HRR})$ as well as \% oxygen uptake reserve $\left(\mathrm{VO}_{2} \mathrm{R}\right)$ are not able to discriminate these phases correctly on an individual basis (Hofmann et al., 2001; Meyer et al., 2005; Scharhag-Rosenberger et al., 2010).

Usually, the first and/or the second turn points (corresponding to the mLaSS) are applied to prescribe exercise intensity limits for prolonged endurance exercise training (Esteve-Lanao et al., 2005; Muñoz et al., 2014a; Tønnessen et al., 2014) whereas the first turn point is rarely investigated (Mann et al., 2013). It is obvious that $\mathrm{LTP}_{2} / \mathrm{VT}_{2}$ clearly discern between sustainable, metabolically balanced or non-sustainable, not metabolically balanced workloads, whereas during exercise near the first turn point a difference in acute responses can only be detected after a very long duration of exercise (Tremblay et al., 2005). Mostly, these small differences in intensity slightly below or above $\mathrm{LTP}_{1} / \mathrm{VT}_{1}$ are not detected and recognized by athletes although it might be important with respect to the particular maximal duration, grade of fatigue and the subsequent recovery time which has been described recently by Burnley and Jones (2016). These authors suggested distinct fatigue mechanisms for each intensity domain. A paucity of fatigue-related mechanistic studies was shown for the moderate and high-intensity domains but lee attention has focused on the low intensity part so far. As can be seen in Figure 3, an intensity slightly above $\mathrm{LTP}_{1}$ already increases La which indicates that the critical lactate clearance rate for the working muscle has been exceeded, and therefore, different hormonal and cardio-respiratory responses are suggested for this intensity level (Moser et al., 2015). In very prolonged exercise with blood lactate remaining at resting level throughout exercise it was prescribed that only after several hours fatigue occurs and increases with time until the limit of tolerance (Burnley and Jones, 2016). It has to be mentioned that energy stores play a substantial role regarding the maximal duration until the point of fatigue (Johnson et al., 2004).

\section{Interval Exercise}

The prescription of intermittent exercise is somehow more complicated compared to continuous exercise as the number of variables is higher. In addition to the workload intensity for the intervals $\left(\mathrm{P}_{\text {peak }}\right)$, the total duration $\left(\mathrm{t}_{\text {total }}\right)$ (number of intervals), the duration of the single workloads $\left(\mathrm{t}_{\text {peak }}\right)$ as well as recovery intensity $\left(\mathrm{P}_{\mathrm{rec}}\right)$ and duration $\left(\mathrm{t}_{\mathrm{rec}}\right)$ and the corresponding mean load $\left(\mathrm{P}_{\text {mean }}\right)$ have to be considered (Buchheit and Laursen, 2013a,b; Tschakert and Hofmann, 2013). Similar to constant load exercise (CLE), the mean intensity and total duration are main markers of the overall workload, but $\mathrm{P}_{\text {mean }}$ is influenced by the 


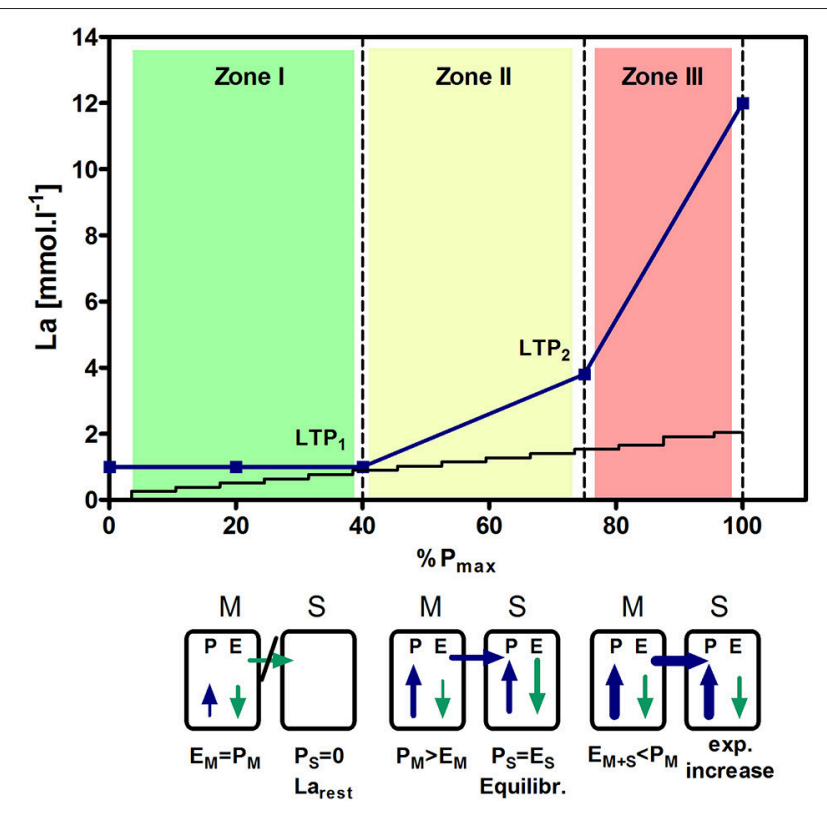

FIGURE 2 | Time course of blood lactate concentration (La) and first $\left(\right.$ LTP $\left._{1}\right)$ and second $\left(\mathbf{L T P}_{2}\right)$ lactate turn points. LTP 1 is the first increase above baseline. Lactate production within the muscle $\left(P_{M}\right)$ is equal to the elimination rate within the working muscle $\left(E_{M}\right)$ and, consequently, no La has to be shuttled to the system (S). La within the system stays at resting level because the critical lactate clearance of the working muscle is not exceeded. This is the first zone which is metabolically balanced at muscle level (M) ( $E_{M}=$ $P_{M}$ ). Above LTP 1 , blood La increases with increasing workloads. La production within the muscle $\left(\mathrm{P}_{\mathrm{M}}\right)$ exceeds the muscular rate of La elimination $\left(E_{M}\right)$ and La has to be shuttled to the system (S). Within the system, La from the muscle $\left(P_{S}\right)$ can be eliminated (by resting muscles, heart, brain,...) $\left(E_{S}+\right.$ $\left.E_{M}=P_{M}\right)$ and La builds up an equilibrium $\left(P_{S}=E_{S}\right)$ at an elevated level, a so called lactate steady state on a systemic level. The critical La clearance rate of the system is not exceeded which gives a metabolically balanced situation (zone II). If the rate of La production within the muscle exceeds the maximal elimination rate of the system and the muscle $\left(P_{M}>E_{M}+E_{S}\right)$, La increases exponentially without a metabolically balanced situation (zone III). The transition from zone II to zone III is indicated by the LTP 2 .

aforementioned variables with respect to the degree and the kind of fatigue and recovery (Burnley and Jones, 2016). Nonetheless, also for intermittent exercise, intensities ( $\left.\mathrm{P}_{\text {peak }}, \mathrm{P}_{\text {rec }}, \mathrm{P}_{\text {mean }}\right)$ are suggested to be set in relation to sub-maximal $\left(\mathrm{LTP}_{1} / \mathrm{VT}_{1}\right.$, $\left.\mathrm{LTP}_{2} / \mathrm{VT}_{2}\right)$ and maximal markers $\left(\mathrm{P}_{\max }\right)$ from an incremental exercise test: $\mathrm{P}_{\text {peak }}=\mathrm{P}_{\text {max }}, \mathrm{P}_{\text {rec }}=\% \mathrm{P}_{\mathrm{LTP} 1}, \mathrm{P}_{\text {mean }}=\% \mathrm{P}_{\mathrm{LTP} 2}$ (Tschakert and Hofmann, 2013).

In addition, we could recently show that aerobic highintensity interval exercise (HIIE) with short workload durations and $\mathrm{P}_{\text {mean-matched constant load exercise produced similar }}$ acute metabolic, hormonal and cardio-respiratory responses (Moser et al., 2015; Tschakert et al., 2015). In contrast, HIIE with long workload durations but the same mean load yielded significantly higher acute physiological responses compared to short HIIE and CLE (Tschakert et al., 2015). This indicated that strictly planning interval-type exercise respecting all variables allows the regulation and the predictability of the acute physiological responses (Tschakert et al., 2015). In a recent paper

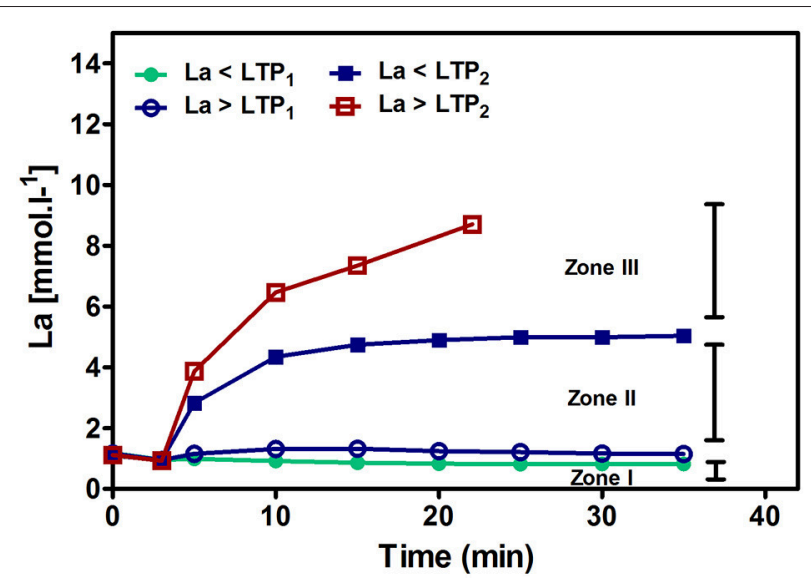

FIGURE 3 | Time course of blood lactate concentration (La) below and above the first $\left(\operatorname{LTP}_{1}\right)$ and the second $\left(\operatorname{LTP}_{2}\right)$ lactate turn points.

Intensities below $\mathrm{LTP}_{1}$ give a lactate steady state at resting level because the critical lactate clearance rate within the working muscle is not exceeded (zone I). For intensities above LTP 2 , some La produced within the muscle is shuttled to the system but La clearance is high enough to establish an equilibrium, the so called systemic La steady state (LaSS) (zone II). Just below $\mathrm{LTP}_{2}$ the maximal lactate clearance rate, the so-called maximal systemic LaSS is approached. Above $\mathrm{LTP}_{2}$, no equilibrium can be established and La increases with time with early termination of exercise due to non-sustainable acidosis (zone III).

we could show that even with a high-intensity running speed, short $10 \mathrm{~s}$ intervals, $20 \mathrm{~s}$ passive recovery but a very low mean load below $\mathrm{LTP}_{1}$, lactate levels were only slightly higher than resting level, and $30 \mathrm{~min}$ of exercise was clearly below the maximal duration which was, however, not obtained in this study (Wallner et al., 2014).

Similar to constant load exercise (Chidnok et al., 2012; SoaresCaldeira et al., 2012), the problem arises how to prescribe the optimal total duration (number of intervals) for intermittent exercise to identify optimal adaptation effects for any specific micro-cycle of a training period (Platonov, 1999; Lyakh et al., 2014). As long as there is a metabolically balanced situation (aerobic interval training), we may treat this problem similar to constant load exercise. In case of increasing La (anaerobic interval training), the optimal number of intervals may be set similar as it is performed in resistance-type exercise (Richens and Cleather, 2014). Again there is urgent need to identify any markers of optimal duration for both constant load and interval-type exercise. Burnley and Jones (2016) highlighted that the power-duration relationship exists not only for constantpower laboratory-based exercise, but also for variable-paced, selfpaced, and intermittent or stochastic exercise, which more closely reflects the "real-world" athletic performance.

\section{PRESCRIPTION OF DURATION}

It is a fact that any certain intensity has its own critical time limit which is dependent on the type of exercise and the kind of athletes, but may be used as an individual diagnostic tool to prescribe exercise duration (Vanhatalo et al., 2011; Pettitt, 


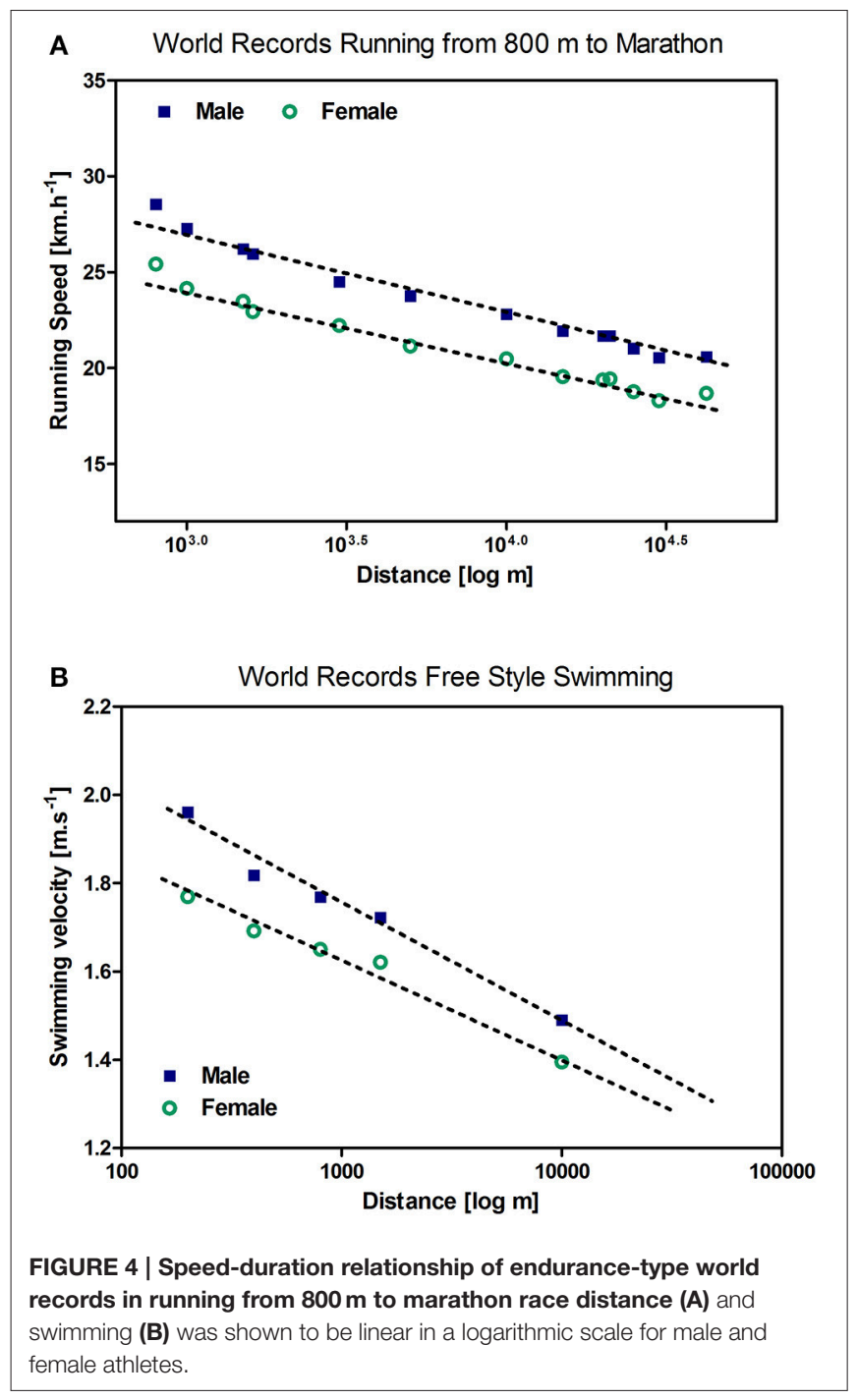

2016; Poole et al., 2016). Figure 4A shows the running speed for all endurance-type world records in continuous running from $800 \mathrm{~m}$ to marathon distance which has been described as a most perfect logarithm relationship for both men and women (Nikolaidis et al., 2017). A similar relationship can be shown for free style swimming (Figure 4B). The relationship between speed and distance is linear applying a logarithmic $\mathrm{X}$-axis within this wide range of race distances. It is obvious that no speeddistance pairs above the linear line are possible (Burnley and Jones, 2016). This speed-distance or power-duration relationship can therefore be applied to detect the maximal speed or power output for any distance or duration but, no less importantly, to detect any maximal duration or distance for an arbitrarily chosen speed or power on an individual basis. Additionally, independent of the chosen intensity, this concept allows setting a targeted duration (\% of maximal duration) for endurance exercise training with respect to improvement, maintenance or recovery purposes. To regulate these distances we apply the concept of Platonov (1999) who differentiated four domains of durations with selective adaptation (Viru, 1995, p. 251). This author suggested "very heavy maximal," "heavy sub-maximal," "moderate," and "low" workloads with respect to duration, but independent of the chosen intensity (Table 1).

Platonov (1999, p. 51) suggested that only "maximal exercise" (75-100\% of the maximal duration until clear fatigue and loss of performance) induces distinct adaptation processes. This maximal exercise needs long recovery of about $48 \mathrm{~h}$ and longer but induces considerable performance increments (Kenttä and Hassmén, 1998; Issurin, 2009) (Figure 5) indicated by hormonal responses and signal-cascades yet not fully understood (Russell et al., 2013; Hoppeler, 2016; Kirby and McCarthy, 2016). Shortening the duration to $60-75 \%$ of the maximal duration until clear fatigue with the same intensity only leads to a compensated fatigue (some signs of fatigue which can be compensated without a loss in performance) which reduces recovery duration to half of the maximal exercise domain (about $24 \mathrm{~h}$ ). The moderate workload is suggested between 20 and $60 \%$ of the maximal duration which does neither induce a compensated nor a clear fatigue and, therefore, does not increase performance but rather stabilizes it. Lastly, low workload defined as duration of less than $20 \%$ of maximal duration (again with the same intensity) induces regeneration and maintains exercise performance. Figure 6 shows an example of the recovery of heart rate variability (HRV) after maximal, sub-maximal, moderate, and low duration exercise with the same intensity applied (unpublished results). HRVwas shown to be sensitive for intensity and duration of exercise (Kaikkonen et al., 2010; Myllymäki et al., 2012).

From this concept (Platonov, 1999, p. 51; Viru, 1995, p. 251), we assume that for any single intensity within the three different intensity domains (Zone I: below $\mathrm{LTP}_{1} / \mathrm{VT}_{1}$; Zone II: between $\mathrm{LTP}_{1} / \mathrm{VT}_{1}$ and $\mathrm{LTP}_{2} / \mathrm{VT}_{2}$; Zone III: above $\mathrm{LTP}_{2} / \mathrm{VT}_{2}$ ), we may chose four different duration domains with clearly distinct adaptation effects on various physiological processes and exercise performance. To prescribe these duration domains one needs the maximal duration for at least 2-3 different intensities to draw the power-duration relationship as can be seen in Figure 7 which shows the same athlete as Figure 1.

\section{Model to Combine the Prescription of Both Intensity and Duration}

Earlier approaches such as the model from Garcin and Billat (2001) applied a perceived exertion scale to attest both intensity and duration, but the optimal duration may not be obtained from this model. As can be seen in Figures 7, 8, the power output (or speed) to time (or distance) relationship allows discerning these specific durations for any intensity of interest by using four different duration domains according to Platonov (in (Viru, 1995), p. 251). As shown in Table 1, zone 1 is defined as low, zone 2 as moderate, zone 3 as sub-maximal, and zone 4 as maximal workload each producing different states of fatigue and, consequently, different effects of adaptation, which is in line with recent data from Tremblay et al. (2005). These authors showed a duration threshold for various hormone responses for a comparable low intensity of $50-55 \% \mathrm{VO}_{2 \max }$ whereas a longer duration induced a favorable hormone profile 
TABLE 1 | Definition of specific duration domains for endurance-type exercise (modified from Platonov, 1999).

\begin{tabular}{|c|c|c|c|}
\hline Workload & Phase & Duration & Targets \\
\hline Low & 1st phase of stable performance & $15-20 \%$ of maximal duration until clear fatigue & Maintaining exercise performance and accelerated recovery \\
\hline Moderate & 2nd phase of stable performance & $20-60 \%$ of maximal duration until clear fatigue & Maintaining exercise performance \\
\hline Sub-maximal & Phase of compensated fatigue & $60-75 \%$ of maximal duration until clear fatigue & Stabilization and moderate increases in performance \\
\hline Maximal & Phase of clear fatigue & $75-100 \%$ of maximal duration until clear fatigue & Distinct increases in performance \\
\hline
\end{tabular}

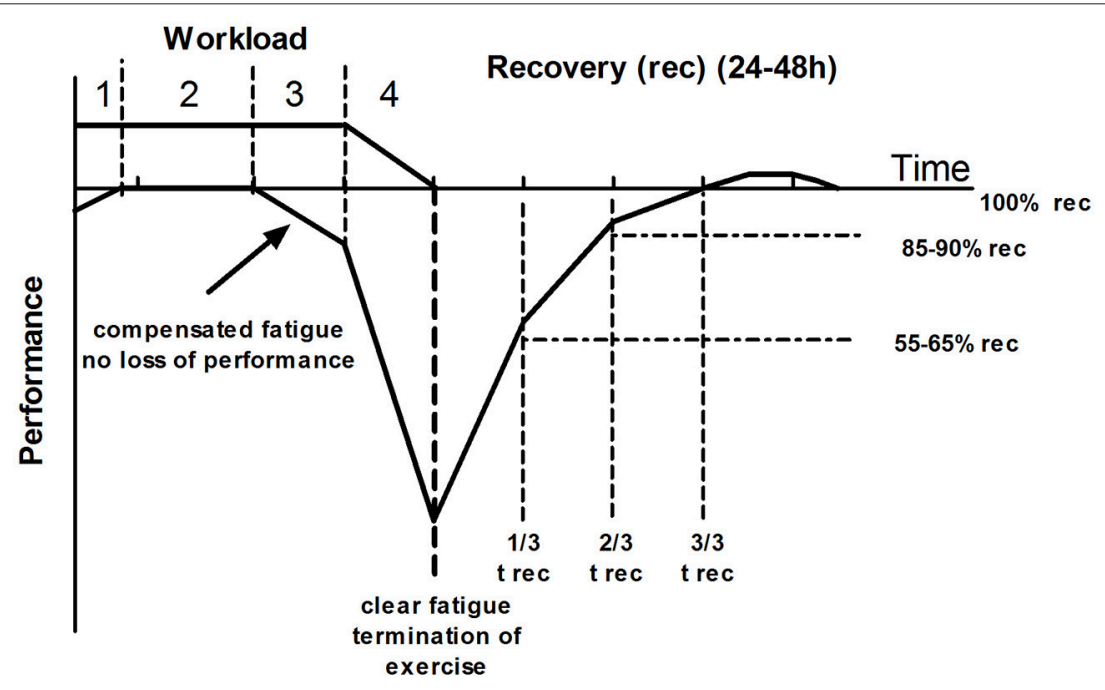

FIGURE 5 | Relationship between duration of exercise and fatigue (modified from Platonov, 1999). There is a certain duration for each intensity leading to distinct grade of fatigue which terminates exercise (4), prolongs recovery times and increases "super-compensation" with increased performance. Reducing the duration to less than $75 \%$ of the maximal duration does not induce distinct but compensated fatigue without a loss in performance (3) and, consequently, a much shorter recovery time and less if any "super-compensation." Reducing duration to less than 60\% of the maximal duration (2) does not induce any fatigue and, therefore, does not increase performance, but rather stabilizes the given performance level. A duration less than $20 \%$ of the maximal duration is just a functional stimulation suggested adequate for regeneration.

which was suggested to support the mobilization of fuels for recovery and restoration of glycogen stores. In this combined exercise prescription model, the setting of work intensities should also be individualized and physiologically based by using turn point intensities as discerning markers for distinctly different metabolic, hormonal and cardio-respiratory responses (Tschakert and Hofmann, 2013; Moser et al., 2015).

This concept (Table 1 and Figure 8) enables athletes and coaches to fine-tune training volume and/or intensity to further optimize training processes which is of particular relevance when the limits of tolerance are reached. In addition, it allows a retrospective analysis of distances covered with given intensities in the past.

\section{DISCUSSION AND CONCLUSIONS}

The concept to combine turn point derived intensities and optimized durations may be specifically interesting with respect to the new polarized training concept (Seiler and Kjerland, 2006; Seiler, 2010; Muñoz et al., 2014a,b; Tønnessen et al., 2014) where $80-90 \%$ of training volume is set below $\mathrm{LTP}_{1} / \mathrm{VT}_{1}$ and up to $22 \%$ above $\mathrm{LTP}_{2} / \mathrm{VT}_{2}$ with very low volumes between both thresholds. It is, however, important to note that some authors also use fixed reference values for lactate such as 2 and 4 mmol.1 $\mathrm{l}^{-1}$ (Seiler and Kjerland, 2006; Guellich et al., 2009; Orie et al., 2014) which may overestimate the volume especially for the low intensity volumes. Our own results showed that $\mathrm{La}$ at $\mathrm{LTP}_{1}$ was found at $1.2-1.6 \mathrm{mmol}^{-1}$ (Hofmann et al., 1997, 2001). An individual and accurate intensity prescription is crucial even at low power outputs near $\mathrm{LTP}_{1}$ since allowing intensities just $10 \%$ above $\mathrm{LTP}_{1}$ definitely shortens the time to clear fatigue by $\sim 40 \%$ (Figure 8). As a consequence, high-volume training set above $\mathrm{LTP}_{1}$ may get too close to a fatigue state that avoids repeating high volumes on a regular daily basis. In addition, for low intensity exercise training, it is usually NOT intended to reach maximal duration $\left(t_{\max }\right)$ but to apply a certain percentage of $t_{\max }$ (Table 1 ) in order to avoid fatigue and to guarantee the ability to repeat high volumes of training on a daily basis. However, we like to point out that dependent on the aim of a specific training period specific types of micro-cycles need to be structured combining exercise type, intensity, and duration.

Beside the attractiveness of the concept, several open questions and limits have to be addressed. Firstly, the chosen 


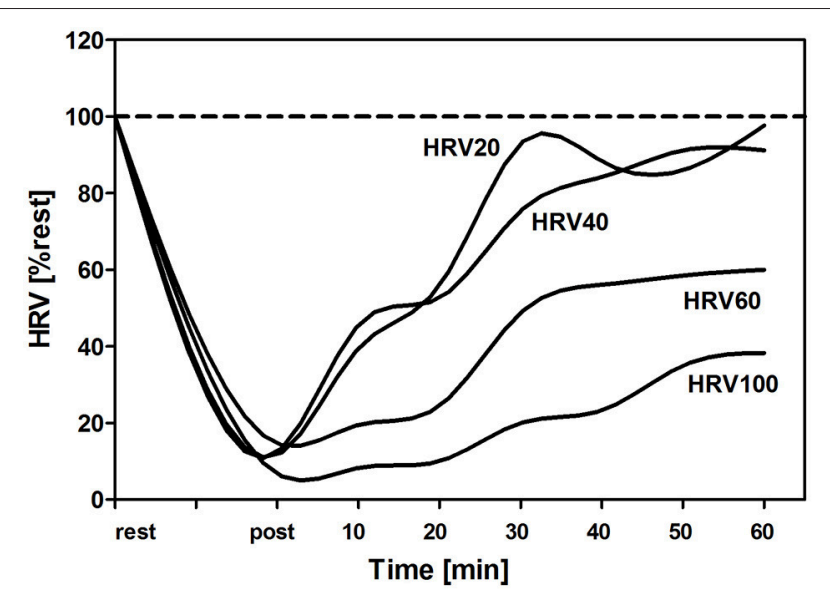

FIGURE 6 | Relative changes of heart rate variability markers (\%HRV) during constant load exercise just below the second lactate turn point with different duration of the maximal sustainable distance $(\mathbf{1 0 0} \%)$. It can be seen that recovery of the HRV is dependent on \% maximal duration with early recovery at low to moderate distances (20,40\%) according to Platonov (1999).

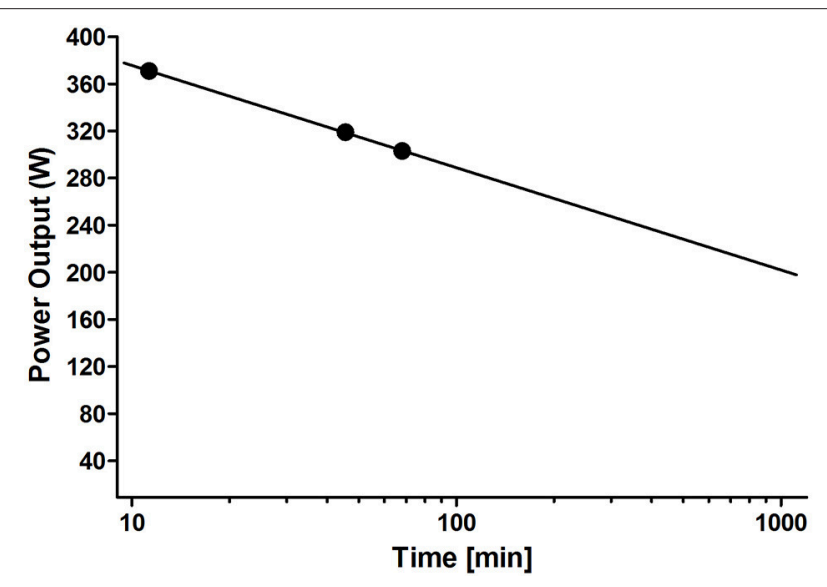

FIGURE 7 | Example of exercise intensity-duration relationship in a single well-trained cyclist. As described in Figure 1, for any individual athlete, a plot of maximal speed and distance relationships can be drawn describing the maximal distance for any specific velocity or power output or the maximal velocity or power output for any chosen distance. According to the modified concept from Platonov (1999), these individual draws allow to prescribe the optimal distance (\% of maximal duration) for selected intensities for various endurance-type training situations. To prescribe both intensity and duration on an individual basis, this concept needs to be combined to the usual sub-maximal performance diagnostic markers such as $\mathrm{LTP}_{1} / \mathrm{VT}_{1}$ and $\mathrm{LTP}_{2} \mathrm{NT}_{2}$.

percentages of maximal duration are just marginally evidenced. To discern the low (regenerative) zone from a moderate zone without fatigue, a zone with compensated fatigue and finally zone 4 with clear fatigue needs to be taken with caution. Carefully conducted studies and retrospective analysis of distances covered at defined intensities are needed to identify the stability or variability of these percentages for athletes with different age,

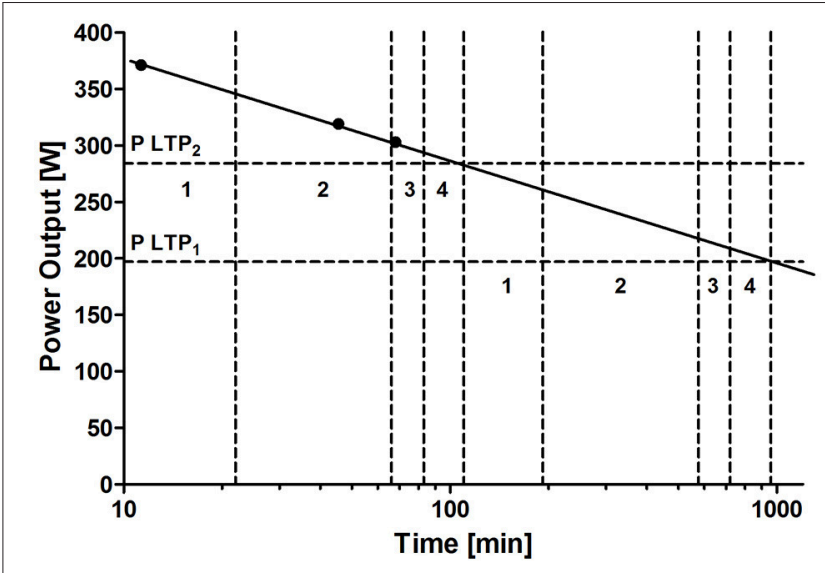

FIGURE 8 | Maximal and optimal (\% of maximal) duration for exercise intensities at LTP $_{1}$ and LTP $_{2}$ in a well-trained cyclist applying the modified concept of Platonov (1999). For any specific metabolic, hormonal or cardio-respiratory target intensity $\left(<\mathrm{LTP}_{1} N \mathrm{NT}_{1}\right.$; between $\mathrm{LTP}_{1} \mathrm{NT}_{1}$ and $\mathrm{LTP}_{2} N \mathrm{~T}_{2}$; $>\mathrm{LTP}_{2} N \mathrm{NT}_{2}$ ), the optimal duration with respect to the four workload domains "low" (1), "moderate" (2), "sub-maximal" (3), and "maximal" (4) may be derived from this graph of the maximal intensity-duration relationship.

training status and sex, and individual physiological markers are needed to guide the training. As could be shown in a pilot test (Figure 6), heart rate variability might be a potential parameter to identify these cut-off points for duration as discussed recently (Kaikkonen et al., 2010; Saboul et al., 2016). Additionally, ratings of perceived exertion (RPE) scales may be helpful to identify these reference markers on a daily individual basis (Garcin and Billat, 2001; Seiler and Sjursen, 2004; Coquart et al., 2012).

A second important limit is the method to derive the power-duration or velocity-distance relationship. To obtain a valid regression line, data points must be obtained from highly motivated athletes from competitions. However, maximal performance changes during the training year due to periodization will make it a bit more difficult to obtain optimal distances throughout the year. Additionally, not all sports allow obtaining these markers under comparable and possibly standardized conditions such as in cycling or on-snow cross-country skiing. Semi-specific tests such as ergometer or ski-roller tests may help to overcome this problem.

A third limit may be the idea to maximize volumes by reducing intensity below $\mathrm{LTP}_{1} / \mathrm{VT}_{1}$. Although athletes may withstand such volumes from an acute metabolic state of view, some long-term problems such as orthopedic complaints (arthrosis, stress fractures) as well as disturbances in energy or fluid supply may arise from such as concept (Noakes, 2000; Cymet and Sinkov, 2006; Krampla et al., 2008; Weber, 2009; Warden et al., 2014).

Despite those limits, this concept gives a solid theoretical framework that allows optimizing both intensity and duration of the whole spectrum of endurance training load for the first time. It may help to improve exercise training for top level performance 
even though it is already close to the limits of tolerance for the human body.

\section{ETHICS STATEMENT}

This methodological consideration included a single pilot tests which were not part of a formal study but a proof of principle determination of markers from standard performance diagnostic tests which was performed in accordance with the

\section{REFERENCES}

Abbiss, C. R., and Laursen, P. B. (2005). Models to explain fatigue during prolonged endurance cycling. Sports Med. 35, 865-898. doi: 10.2165/00007256-200535100-00004

Algrøy, E. A., Hetlelid, K. J., Seiler, S., and Stray Pedersen, J. I. (2011). Quantifying training intensity distribution in a group of Norwegian professional soccer players. Int. J. Sports Physiol. Perform. 6, 70-81. doi: 10.1123/ijspp.6.1.70

Amann, M., Subudhi, A., and Foster, C. (2004). Influence of testing protocol on ventilatory thresholds and cycling performance. Med. Sci. Sports Exerc. 36, 613-622. doi: 10.1249/01.MSS.0000122076.21804.10

Brickley, G., Doust, J., and Williams, C. A. (2002). Physiological responses during exercise to exhaustion at critical power. Eur. J. Appl. Physiol. 88, 146-151. doi: 10.1007/s00421-002-0706-1

Brooks, G. A. (1986). The lactate shuttle during exercise and recovery. Med. Sci. Sports Exerc. 18, 360-368. doi: 10.1249/00005768-198606000-00019

Brooks, G. A. (2009). Cell-cell and intracellular lactate shuttles. J. Physiol. 587(Pt 23), 5591-5600. doi: 10.1113/jphysiol.2009.178350

Buchheit, M., and Laursen, P. B. (2013a). High-intensity interval training, solutions to the programming puzzle: part I: cardiopulmonary emphasis. Sports Med. 43, 313-338. doi: 10.1007/s40279-013-0029-x

Buchheit, M., and Laursen, P. B. (2013b). High-intensity interval training, solutions to the programming puzzle. Part II: anaerobic energy, neuromuscular load and practical applications. Sports Med. 43, 927-954. doi: $10.1007 /$ s40279-013-0066-5

Burnley, M., and Jones, A. M. (2016). Power-duration relationship: physiology, fatigue, and the limits of human performance. Eur. J. Sport Sci. 3, 1-12. doi: 10.1080/17461391.2016.1249524

Chidnok, W., Dimenna, F. J., Bailey, S. J., Vanhatalo, A., Morton, R. H., Wilkerson, D. P., et al. (2012). Exercise tolerance in intermittent cycling: application of the critical power concept. Med. Sci. Sports Exerc. 44, 966-976. doi: 10.1249/MSS.0b013e31823ea28a

Coquart, J. B., Eston, R. G., Noakes, T. D., Tourny-Chollet, C., L'hermette, M., Lemaître, F., et al. (2012). Estimated time limit: a brief review of a perceptually based scale. Sports Med. 42, 845-855. doi: 10.2165/11635370-000000000-00000

Cymet, T. C., and Sinkov, V. (2006). Does long-distance running cause osteoarthritis? J. Am. Osteopath. Assoc. 106, 342-345.

Dekerle, J., Baron, B., Dupont, L., Vanvelcenaher, J., and Pelayo, P. (2003). Maximal lactate steady state, respiratory compensation threshold and critical power. Eur. J Appl. Physiol. 89, 281-288. doi: 10.1007/s00421-002-0786-y

Dittrich, N., de Lucas, R. D., Beneke, R., and Guglielmo, L. G. (2014). Time to exhaustion at continuous and intermittent maximal lactate steady state during running exercise. Int. J. Sports Physiol. Perform. 9, 772-776. doi: 10.1123/ijspp.2013-0403

Esteve-Lanao, J., San Juan, A. F., Earnest, C. P., Foster, C., and Lucia, A. (2005). How do endurance runners actually train? Relationship with competition performance. Med. Sci. Sports Exerc. 37, 496-504. doi: 10.1249/01.MSS.0000155393.78744.86

Faude, O., Hecksteden, A., Hammes, D., Schumacher, F., Besenius, E., Sperlich, B., et al. (2017). Reliability of time-to-exhaustion and selected psycho-physiological variables during constant-load cycling at the maximal lactate steady-state. Appl. Physiol. Nutr. Metab. 42, 142-147. doi: 10.1139/apnm-2016-0375

Fontana, P., Boutellier, U., and Knöpfli-Lenzin, C. (2009). Time to exhaustion at maximal lactate steady state is similar for cycling and running recommendations of Declaration of Helsinki. The subject gave written informed consent in accordance with the Declaration of Helsinki.

\section{AUTHOR CONTRIBUTIONS}

PH: Author wrote the manuscript, draw the figures, and graphs. GT: Author contributed equally in writing the manuscript.

in moderately trained subjects. Eur. J. Appl. Physiol. 107, 187-192. doi: 10.1007/s00421-009-1111-9

Garcin, M., and Billat, V. (2001). Perceived exertion scales attest to both intensity and exercise duration. Percept. Mot. Skills 93, 661-671. doi: 10.2466/pms.2001.93.3.661

Guellich, A., Seiler, S., and Emrich, E. (2009). Training methods and intensity distribution of young world-class rowers. Int. J. Sports. Physiol. Perform. 4, 448-460. doi: 10.1123/ijspp.4.4.448

Hofmann, P., Pokan, R., von Duvillard, S. P., Seibert, F. J., Zweiker, R., and Schmid, P. (1997). Heart rate performance curve during incremental cycle ergometer exercise in healthy young male subjects. Med. Sci. Sports Exerc. 29, $762-768$.

Hofmann, P., and Tschakert, G. (2010). Special needs to prescribe exercise intensity for scientific studies. Cardiol. Res. Pract. 2011:209302. doi: 10.4061/2011/209302

Hofmann, P., Von Duvillard, S. P., Seibert, F. J., Pokan, R., Wonisch, M., Lemura, L. M., et al. (2001). \%HRmax target heart rate is dependent on heart rate performance curve deflection. Med. Sci. Sports Exerc. 33, 1726-1731. doi: 10.1097/00005768-200110000-00017

Hoppeler, H. (2016). Molecular networks in skeletal muscle plasticity. J. Exp. Biol. 219 (Pt 2), 205-213. doi: 10.1242/jeb.128207

Issurin, V. B. (2009). Generalized training effects induced by athletic preparation. A review. J. Sports Med. Phys. Fitness 49, 333-345.

Johnson, N. A., Stannard, S. R., and Thompson, M. W. (2004). Muscle triglyceride and glycogen in endurance exercise: implications for performance. Sports Med. 34, 151-164. doi: 10.2165/00007256-200434030-00002

Jones, A. M., Vanhatalo, A., Burnley, M., Morton, R. H., and Poole, D. C. (2010). Critical power: implications for determination of VO2max and exercise tolerance. Med. Sci. Sports Exerc. 42, 1876-1890. doi: 10.1249/MSS.0b013e3181d9cf7f

Jones, A. M., Wilkerson, D. P., DiMenna, F., Fulford, J., and Poole, D. C. (2008). Muscle metabolic responses to exercise above and below the "critical power" assessed using 31P-MRS. Am. J. Physiol. Regul. Integr. Comp. Physiol. 294, R585-R593. doi: 10.1152/ajpregu.00731.2007

Kaikkonen, P., Hynynen, E., Mann, T., Rusko, H., and Nummela, A. (2010). Can HRV be used to evaluate training load in constant load exercises? Eur. J. Appl. Physiol. 108, 435-442. doi: 10.1007/s00421-009-1240-1

Kenttä, G., and Hassmén, P. (1998). Overtraining and recovery. A conceptual model. Sports Med. 26, 1-16. doi: 10.2165/00007256-199826010-00001

Kirby, T. J., and McCarthy, J. J. (2016). MicroRNAs in skeletal muscle biology and exercise adaptation. Free Radic. Biol. Med. 64, 95-105. doi: 10.1016/j.freeradbiomed.2013.07.004

Krampla, W. W., Newrkla, S. P., Kroener, A. H., and Hruby, W. F. (2008). Changes on magnetic resonance tomography in the knee joints of marathon runners: a 10-year longitudinal study. Skeletal. Radiol. 37, 619-626. doi: 10.1007/s00256-008-0485-9

Lundby, C., and Robach, P. (2015). Performance Enhancement: what are the physiological limits? Physiology 30, 282-292. doi: 10.1152/physiol.00052.2014

Lyakh, V., Mikołajec, K., Bujas, P., and Litkowycz, R. (2014). Review of platonov's "sports training periodization. general theory and its practical application" - kiev: olympic literature, 2013. J. Hum. Kinet. 30, 259-263. doi: 10.2478/hukin-2014-0131

Mann, T., Lamberts, R. P., and Lambert, M. I. (2013). Methods of prescribing relative exercise intensity: physiological and practical considerations. Sports Med. 43, 613-625. doi: 10.1007/s40279-013-0045-x 
McLellan, T. M. (1985). Ventilatory and plasma lactate response with different exercise protocols: a comparison of methods. Int. J. Sports Med. 6, 30-35. doi: 10.1055/s-2008-1025809

Meyer, T., Lucía, A., Earnest, C. P., and Kindermann, W. (2005). A conceptual framework for performance diagnosis and training prescription from submaximal gas exchange parameters-theory and application. Int. J. Sports Med. 26(Suppl. 1), S38-S48. doi: 10.1055/s-2004-830514

Moser, O., Tschakert, G., Mueller, A., Groeschl, W., Pieber, T. R., ObermayerPietsch, B., et al. (2015). Effects of high-intensity interval exercise versus moderate continuous exercise on glucose homeostasis and hormone response in patients with type 1 diabetes mellitus using novel ultra-long-acting insulin. PLOS ONE 10:e0136489. doi: 10.1371/journal.pone.0136489

Muñoz, I., Cejuela, R., Seiler, S., Larumbe, E., and Esteve-Lanao, J. (2014a). Training-intensity distribution during an ironman season: relationship with competition performance. Int. J. Sports Physiol. Perform. 9, 332-339. doi: 10.1123/ijspp.2012-0352.

Muñoz, I., Seiler, S., Bautista, J., España, J., Larumbe, E., and Esteve-Lanao, J. (2014b). Does polarized training improve performance in recreational runners? Int. J. Sports Physiol. Perform. 9, 265-272. doi: 10.1123/ijspp.2012-0350

Myllymäki, T., Rusko, H., Syväoja, H., Juuti, T., Kinnunen, M. L., and Kyröläinen, H. (2012). Effects of exercise intensity and duration on nocturnal heart rate variability and sleep quality. Eur J Appl Physiol. 112, 801-809. doi: 10.1007/s00421-011-2034-9

Nikolaidis, P. T., Zingg, M. A., and Knechtle, B. (2017). Performance trends in age-group runners from $100 \mathrm{~m}$ to marathon-The World Championships from 1975 to 2015. Scand. J. Med. Sci. Sports doi: 10.1111/sms.12821. [Epub ahead of print].

Noakes, T. D. (2000). Physiological models to understand exercise fatigue and the adaptations that predict or enhance athletic performance. Scand. J. Med. Sci. Sports. 10, 123-145. doi: 10.1034/j.1600-0838.2000.010003123.x

Orie, J., Hofman, N., de Koning, J. J., and Foster, C. (2014). Thirty-eight years of training distribution in Olympic speed skaters. Int. J. Sports Physiol. Perform. 9, 93-99. doi: 10.1123/ijspp.2013-0427

Périard, J. D., Caillaud, C., and Thompson, M. W. (2012). The role of aerobic fitness and exercise intensity on endurance performance in uncompensable heat stress conditions. Eur. J. Appl. Physiol. 112, 1989-1999. doi: $10.1007 / \mathrm{s} 00421-011-2165-\mathrm{z}$

Pescatello, L. S. (ed.) (2014). ACSM's Guidelines for Exercise Testing and Prescription, 9th Edn. Baltimore, MD: Wolters Kluwer-Lippincott Williams $\&$ Wilkins.

Pettitt, R. W. (2016). Applying the critical speed concept to racing strategy and interval training prescription. Int. J. Sports Physiol. Perform. 11, 842-847. doi: 10.1123/ijspp.2016-0001

Platonov, V. N. (1999). Workload - Fatigue - Performance: The modern framework of exercise training. (Belastung - Ermüdung - Leistung: Der moderne Trainingsaufbau). Münster: Phillippka Sportverlag (german).

Pokan, R., Ocenasek, H., Hochgatterer, R., Miehl, M., Vonbank, K., Von Duvillard, S. P., et al. (2014). Myocardial dimensions and hemodynamics during 24-h ultraendurance ergometry. Med. Sci. Sports Exerc. 46, 268-275. doi: 10.1249/MSS.0b013e3182a64639

Poole, D. C., Burnley, M., Vanhatalo, A., Rossiter, H. B., and Jones, A. M. (2016). Critical power: an important fatigue threshold in exercise physiology. Med. Sci. Sports Exerc. 48, 2320-2334. doi: 10.1249/MSS.0000000000000939

Pringle, J. S., and Jones, A. M. (2002). Maximal lactate steady state, critical power and EMG during cycling. Eur. J. Appl. Physiol. 88, 214-226. doi: 10.1007/s00421-002-0703-4

Richens, B., and Cleather, D. J. (2014). The relationship between the number of repetitions performed at given intensities is different in endurance and strength trained athletes. Biol. Sport. 31, 157-161. doi: 10.5604/20831862.1099047

Russell, A. P., Lamon, S., Boon, H., Wada, S., Güller, I., Brown, E. L., et al. (2013). Regulation of miRNAs in human skeletal muscle following acute endurance exercise and short-term endurance training. J. Physiol. 591, 4637-4653. doi: 10.1113/jphysiol.2013.255695

Saboul, D., Balducci, P., Millet, G., Pialoux, V., and Hautier, C. (2016). A pilot study on quantification of training load: the use of HRV in training practice. Eur. J. Sport Sci. 16, 172-181. doi: 10.1080/17461391.2015.1004373

Scharhag-Rosenberger, F., Meyer, T., Gässler, N., Faude, O., and Kindermann, W. (2010). Exercise at given percentages of VO2max: heterogeneous metabolic responses between individuals. J. Sci. Med. Sports 13, 74-79. doi: 10.1016/j.jsams.2008.12.626

Seiler, K. S., and Kjerland, G. Ø. (2006). Quantifying training intensity distribution in elite endurance athletes: is there evidence for an "optimal" distribution? Scand. J. Med. Sci. Sports 16, 49-56. doi: 10.1111/j.1600-0838.2004. 00418.x

Seiler, S. (2010). What is best practice for training intensity and duration distribution in endurance athletes? Int. J. Sports Physiol. Perform. 5, 276-291. doi: 10.1123/ijspp.5.3.276

Seiler, S., and Sjursen, J. E. (2004). Effect of work duration on physiological and rating scale of perceived exertion responses during self-paced interval training. Scand. J. Med. Sci. Sports 14, 318-325. doi: 10.1046/j.1600-0838.2003. 00353.x

Skovgaard, C., Brandt, N., Pilegaard, H., and Bangsbo, J. (2016). Combined speed endurance and endurance exercise amplify the exercise-induced PGC- $1 \alpha$ and PDK4 mRNA response in trained human muscle. Physiol. Rep. 4:e12864. doi: 10.14814 /phy2.12864

Soares-Caldeira, L. F., Okuno, N. M., Magalhães Sales, M., Campbell, C. S. Simões, H. G., and Nakamura, F. Y. (2012). Similarity in physiological and perceived exertion responses to exercise at continuous and intermittent critical power. Eur. J. Appl. Physiol. 112, 1637-1644. doi: 10.1007/s00421-0112123-9

Stöggl, T. L., and Sperlich, B. (2015). The training intensity distribution among well-trained and elite endurance athletes. Front. Physiol. 6:295. doi: 10.3389/fphys.2015.00295

Tønnessen, E., Svendsen, I. S., Rønnestad, B. R., Hisdal, J., Haugen, T. A., and Seiler, S. (2015). The annual training periodization of 8 world champions in orienteering. Int. J. Sports Physiol. Perform. 10, 29-38. doi: 10.1123/ijspp.2014-0005

Tønnessen, E., Sylta, Ø., Haugen, T. A., Hem, E., Svendsen, I. S., and Seiler, S. (2014). The road to gold: training and peaking characteristics in the year prior to a gold medal endurance performance. PLOS ONE 9:e101796. doi: 10.1371/journal.pone.0101796

Tremblay, M. S., Copeland, J. L., and Van Helder, W. (2005). Influence of exercise duration on post-exercise steroid hormone responses in trained males. Eur. J. Appl. Physiol. 94, 505-513. doi: 10.1007/s00421-005-1380-x

Tschakert, G., and Hofmann, P. (2013). High-intensity intermittent exercise: methodological and physiological aspects. Int. J. Sports Physiol. Perform. 8, 600-610. doi: 10.1123/ijspp.8.6.600

Tschakert, G., Kroepfl, J., Mueller, A., Moser, O., Groeschl, W., and Hofmann, P. (2015). How to regulate the acute physiological response to "aerobic" high-intensity interval exercise. J. Sports Sci. Med. 14, 29-36.

Vanhatalo, A., Jones, A. M., and Burnley, M. (2011). Application of critical power in sport. Int. J. Sports Physiol. Perform. 6, 128-136. doi: 10.1123/ijspp.6.1.128

Viru, A. (1995). Adaptation in Sports Training. Boca Raton, FL: CRC Press.

Viru, A., Smirnova, T., Karelson, K., Snegovskaya, V., and Viru, M. (1996). Determinants and modulators of hormonal responses to exercise. Biol. Sports 13, 169-187.

Wallner, D., Simi, H., Tschakert, G., and Hofmann, P. (2014). Acute physiological response to aerobic short-interval training in trained runners. Int. J. Sports Physiol. Perform. 9, 661-666. doi: 10.1123/ijspp.2013-0385

Warden, S. J., Davis, I. S., and Fredericson, M. (2014). Management and prevention of bone stress injuries in long-distance runners. J. Orthop. Sports Phys. Ther. 44, 749-765. doi: 10.2519/jospt.2014.5334

Weber, J. M. (2009). The physiology of long-distance migration: extending the limits of endurance metabolism. J. Exp. Biol. 212 (Pt 5), 593-597. doi: $10.1242 /$ jeb.015024

Conflict of Interest Statement: The authors declare that the research was conducted in the absence of any commercial or financial relationships that could be construed as a potential conflict of interest.

Copyright (C) 2017 Hofmann and Tschakert. This is an open-access article distributed under the terms of the Creative Commons Attribution License (CC BY). The use, distribution or reproduction in other forums is permitted, provided the original author(s) or licensor are credited and that the original publication in this journal is cited, in accordance with accepted academic practice. No use, distribution or reproduction is permitted which does not comply with these terms. 